\title{
Evaluation de la consommation d'un troupeau de chèvres laitières sur parcours forestier \\ - Méthode d'observation directe des coups de dents - Méthode du marqueur oxyde de chrome
}

\author{
M. MEURET * (1), Nicole BARTIAUX-THILL * et A. BOURBOUZE *** \\ avec la collaboration technique de Sophie Rosenberger ****, Marie Vernerey ****, \\ Y. Sourbier $* * * *$, Véronique Ninane $* * * * *$, Michèle, Marianne Trojan $* * * * *$, \\ N. ROUCHY et J.-F. ANDRÉ ****** \\ * Université Libre de Bruxelles, Section interfacultaire d'Agronomie \\ Laboratoire de Botanique systématique et d'Ecologie \\ Ministère de l'Agriculture de Belgique, Administration de la Recherche agronomique \\ ** Centre de Recherche agronomique de l'Etat, Station de Zootechnie \\ $B 5800$ Liroux-Gembloux \\ *** Institut national agronomique Paris-Grignon, Chaire de Zootechnie \\ 16, rue Claude-Bernard, $F 75231$ Paris Cedex 05 \\ ***** Association «Le Mat», Elevage caprin du Viel Audon \\ F 07120 Balazuc \\ *****: Université Libre de Bruxelles, Section interfacultaire d'Agronomie \\ avenue Paul-Héger, B 1050 Bruxelles
}

\begin{abstract}
Résumé
Deux méthodes d'estimation de la quantité de matière sèche ingérée par des chèvres au pâturage sur parcours sont étudiées. Pour cela, elles sont appliquées simultanément durant 6 jours à 5 chèvres laitières de race Alpine chamoisée pâturant un taillis de chêne blanc (Quercus pubescens).

Lc suivi par observation directe des coups de dents a été réalisé à raison de 10 minutes de relevé par demi-heure de pâturage. L'estimation du «poids du coup de dents » porté sur les espèces ligneuses ( 75 p. 100 en poids de la ration) a été effectuée sur les sites de consommation, à l'aide de distribution de rameaux pesés sur balance de précision.

Le marqueur oxyde de chrome-papier, incorporé à du foin de luzerne pressé en pellets, a été ingéré sans pertes ni refus. Le dosage de l'oxyde de chrome a été réalisé par titrage après oxydation nitro-pcrchlorique. Une courbe d'excrétion fécale du $\mathrm{Cr}_{2} \mathrm{O}_{3}$ par des caprins sur parcours a été établie.

Les quantités ingérées (exprimées en $\mathrm{kg}$ MS/animal.jour) sont respectivement pour la méthode des coups de dents et pour la méthode oxyde de chrome de 2,61 et 2,16. Ces résultats montrent qu'une utilisation importante du parcours $(82$ p. 100 des apports énergétiques totaux), liée à une faible valeur alimentaire de la ration ligneuse du parcours (0,59 UFL/kg MS) conduit à un niveau de consommation totale quotidienne assez élevé (voisin de $2,7 \mathrm{~kg} \mathrm{MS} /$ jour pour un animal de $60 \mathrm{~kg} \mathrm{PV}$ ).
\end{abstract}

(1) Adresse actuelle : I.N.R.A., Centre de Recherches d'Avignon, Unité d'Ecodéveloppement, Groupe de Recherche de Provence, Domaine Saint-Paul, 84140 Montfavet (France). 
L'application des méthodes fait apparaître des incertitudes liées à l'insuffisance des connaissances relatives au sujet traité (digestibilité réelle de la ration, supplément de besoin d'entretien dû aux déplacements). La discussion des problèmes rencontrés souligne la nécessité de poursuivre les recherches sur ce type de pâturage.

Mots clés : chèvre, parcours, quantité ingérée, méthode comportementale, marqueur indigestible, oxyde de chrome, comportement alimentaire.

\section{Introduction}

Différents facteurs régissent le mode d'utilisation des parcours par les ruminants. Parmi ceux-ci, la quantité de fourrage consommé est un élément souvent délicat à déterminer, notamment sur les parcours forestiers où les caprins utilisent différentes strates de la végétation.

Aucune méthode ne donne pleinement satisfaction. Classiquement, on distingue les méthodes directes mesurant la production primaire et les méthodes indirectes sur l'animal (MeıJs, 1981). Parmi ces dernières, les plus utilisées sont les techniques utilisant les marqueurs, les techniques index fécal et les techniques utilisant les fistules œsophagiennes. Les méthodes par observation directe du comportement animal sont encore peu utilisées au pâturage à cause de leur imprécision malgré les avantages qu'elles présentent : utilisation possible pour des estimations hors station sur des parcours hétérogènes, mesures simples et rapidité de mise en œuvre, analyses de laboratoire réduites au minimum (ARNOLD et DUDZINSKI, 1967 ; ALLDEN \& WhitTAKER, 1970 ; CHACON et al., 1976).

Sans trop vouloir comparer des méthodes qui procèdent de démarches très différentes, il nous a paru intéressant de tester en parallèle la technique de l'observation directe des coups de dents, adaptée pour la circonstance, et la méthode du marqueur indigestible oxyde de chrome, afin d'apprécier les avantages et les inconvénients respectifs de ces deux approches.

L'expérience a été réalisée lors de l'exploitation estivale d'un taillis de chêne blanc (Quercus pubescens) par un troupeau de chèvres laitières.

\section{Matériel et méthodes}

\section{A. Conduite du troupeau}

Le troupeau suivi est composé de 30 chèvres laitières de race Alpine chamoisée. La production laitière annuelle par animal est de 580 litres (260 jours de lactation). La contribution annuelle globale des parcours aux apports énergétiques du troupeau est supérieure à 70 p. 100 des apports totaux. Les chèvres sont gardées en majeure partie sur un parcours forestier (110 ha). Ce dernier, situé à 200 mètres d'altitude sur les plateaux calcaires en bordure de la rivière l'Ardèche, est composé exclusivement de taillis de chêne blanc (Quercus pubescens) et de clairières à brôme érigé (Bromus erectus) et psoralée bitumineuse (Psoralea bituminosa). 
Durant l'été, l'absence de points d'eau sur les plateaux conduit à limiter très fortement la superficie de la zone utilisée ( $23 \mathrm{ha}$ ). L'expérience, effectuée du 25 juillet au 10 août 1983, a respecté l'horaire de sortie estivale des animaux : 7 h 30 $11 \mathrm{~h} 30$ et $18 \mathrm{~h} 00-21 \mathrm{~h} 30$, soit $7 \mathrm{~h} 30$ de sortie par jour, dont $6 \mathrm{~h}$ de pâturage.

\section{B. Animaux et alimentation}

Dans le but d'appliquer simultanément les deux méthodes d'estimation des quantités ingérées, on choisit 5 animaux témoins de plus d'un an, représentatifs de l'ensemble du troupeau producteur. Leurs caractéristiques zootechniques principales sont présentées au tableaut 4. Ces animaux sont d'une part marqués à l'oxyde de chrome et d'autre part suivis par la méthode d'observation directe des coups de dents. Au moment de l'essai, les animaux reçoivent un complément énergétique de $0,35 \mathrm{~kg}$ $\mathrm{MS} /$ jour . animal, correspondant à 20 p. 100 de leur besoin théorique moyen. L'essentiel de leur alimentation est donc prélevé sur le parcours.

\section{Méthode d'observation directe des coups de dents}

La méthode est basée sur l'observation directe du comportement des animaux sur parcours et le comptage des coups de dents qu'ils portent sur les différentes espèces végétales (bite count).

En principe, cette méthode n'est utilisée que pour l'estimation de la composition botanique de la ration d'animaux domestiques ou sattvages (NEFF, 1974 ; GrIEGO, 1975 ; Currie et al., 1977). Plus récemment, certains auteurs se sont efforcés de quantifier l'ingéré par la mesure du poids du coup de dents (STOBbS, 1975 ; Le Du \& Penning, 1979 ; Bourbouze, 1980).

Le «coup de dents" correspond, chez le caprin, à un ou plusieurs coups de langue suivis d'un retrait de la tête sectionnant la végétation pincée entre les incisives inféricures et le bourrelet gingival supérieur. L'animal doit être observé à quelques mètres afin de faciliter le comptage des coups de dents; cela nécessite l'accoutumance des animaux à la présence d'un observateur. Ce dernier note la fréquence de coups de dents observée sur chacune des espèces consommées (exprimée en $\mathrm{CD} /$ minute de pâturage), et il évalue, par ailleurs, la quantité de MS ingérée par un coup de dents porté sur ces espèces (exprimée en $\mathrm{g} / \mathrm{CD}$ ).

On a :

$$
\mathrm{MSI}=\frac{\mathrm{DP}}{\mathrm{R}} \sum_{\mathrm{i}}\left(\mathrm{CD}_{\mathrm{i}} \cdot \mathrm{PCD}_{\mathrm{i}}\right)
$$

avec :

MSI = matière sèche ingérée au pâturage,

$\mathrm{DP}=$ durée totale de pâturage,

$\mathrm{R}=$ durée totale des périodes de comptage des coups de dents,

$\mathrm{CD}_{\mathrm{i}}=$ nombre total de coups de dents portés sur l'espèce $\mathrm{i}$ durant les périodes de comptage,

$\mathrm{PCD}_{\mathrm{i}}=$ poids du coup de dents moyen porté sur l'espèce $\mathrm{i}$. 
L'évaluation du poids unitaire d'un coup de dents porté sur une espèce donnée est pratiquée suivant deux méthodes distinctes, selon qu'il s'agit d'une espèce arbustive ou d'une espèce herbacée. Cette évaluation est réalisée à des périodes voisines des relevés de la fréquence de coups de dents.

\section{Espèces arbustives}

La méthode consiste à faire donner, par des animaux choisis au hasard dans le troupeau, un certain nombre de coups de dents sur un rameau présenté à bout de bras par l'expérimentateur. Cette distribution, opérée au sein même de la végétation arbustive, tente de reproduire des conditions de broutage aussi naturelles que possible. Le rameau est pesé une première fois intact, puis une seconde fois, en partie ou presque totalement consommé. Les pesées sont réalisées à l'aide d'une balance de précision (type Mettler $-\mathrm{d}=0,2 \mathrm{~g}$ ) fonctionnant sur batterie, sur le site même des parcours, à raison de 4 séances par jour, réparties sur l'ensemble de la période de sortie. Il a été comptabilisé ainsi de 200 à 2000 coups de dents par espèce, sur un certain nombre de rameaux.

On a :

$$
\mathrm{PCD}=\frac{\mathrm{P}_{\mathrm{i}}-\mathrm{P}_{\mathrm{f}}}{\mathrm{n}}
$$

avec :

PCD = poids moyen du coup de dents porté sur le rameau,

$\mathbf{P}_{\mathrm{i}} \quad=$ poids initial du rameau,

$\mathrm{P}_{\mathrm{f}} \quad=$ poids final du rameau,

$\mathrm{n} \quad=$ nombre de coups de dents portés sur le rameau $(\mathrm{n}=10$ à 30$)$.

\section{Espèces herbacées}

Suite à une observation fine du mode de prélèvement de l'espèce par les animaux (réalisée lors des relevés de la fréquence de coups de dents), on effectue 100 à 500 prélèvements par pinçage manuel des quantités représentatives d'un coup de dents (hand clipping). Cette simulation est doublement facilitée par le fait que les caprins ne prélèvent en général qu'une espèce à la fois et que la densité de la strate herbacée est faible.

Le rythme d'observation de la fréquence de coups de dents doit être choisi en fonction du degré d'hétérogénéité du milieu pâturé. Dans le cas présent, les animaux sont observés à raison de 10 minutes de relevé par 30 minutes de pâturage.

On entend par «pâturage », le "pâturage vrai » (où l'animal se déplace à très faible vitesse - entre 0 et $0,1 \mathrm{~m} / \mathrm{s}$ - et consomme sans interruption) et le "pâturage-déplacement » (où la vitesse de déplacement - voisine de $0,3 \mathrm{~m} / \mathrm{s}$ - permet encore à l'animal de brouter significativement). C'est sur l'ensemble de ces deux types d'activité que les relevés systématiques de la fréquence de coups de dents sont réalisés. Seul a été exclu des relevés le troisième type d'activité (la " marche forcée »), où une vitesse de déplacement trop importante (voisine de $0,8 \mathrm{~m} / \mathrm{s}$ ) conduit à une consommation proche de zéro.

On estime ainsi la composition botanique de la ration (exprimée en p. 100 du poids sec des différentes espèces consommées) calculée sur l'ensemble des séquences 
contrôlées. On mesure en outre la durée totale des périodes de relevés (R) et la durée totale de pâturage (DP).

\section{Méthode du marqueur oxyde de chrome}

L'oxyde de chrome $\left(\mathrm{Cr}_{2} \mathrm{O}_{3}\right)$, marqueur externe de la phase solide des digesta, est très utilisé dans les études de digestion. ThILl et al. (1978) ont montré qu'il n'est pas absorbé et que, administré sous une forme appropriée (papier à l'oxyde de chrome), son comportement au cours du transit est fort semblable à celui du radiocérium, considéré par de nombreux auteurs comme le marqueur idéal.

Dans notre essai, l'oxyde de chrome est administré quotidiennement (au moment des complémentations en grain : $6 \mathrm{~h}$ et $17 \mathrm{~h}$ ) durant les 7 jours pré-expérimentaux nécessaires à l'établissement d'un équilibre de transit, et durant les 6 jours de prélèvement des matières fécales. Il est distribué en 2 doses de $2 \mathrm{~g} /$ animal, mélangées chacune à $60 \mathrm{~g}$ de luzerne déshydratée présentée sous forme de pellets ${ }^{(2)}$.

Les matières fécales sont récoltées durant la totalité de la phase diurne d'activité des animaux $(5 \mathrm{~h}-22 \mathrm{~h})$, à raison de 8 échantillons en moyenne par jour et par animal, répartis de manière homogène sur l'ensemble de la journée. Cela afin d'établir une courbe d'excrétion fécale du marqueur oxyde de chrome dans le cas de caprins sur parcours.

La difficulté d'adapter à des caprins les sacs collecteurs de fèces proposés par Tissier et al. (1975) (3) nous a conduit à adopter la méthode du prélèvement à l'aide d'un récipient tenu à bout de bras par l'expérimentateur. Cette technique, relativement souple d'utilisation tant sur parcours qu'à l'étable, pratiquée simultanément avec le relevé de la fréquence de coups de dents, a permis d'effectuer les prélèvements fécaux sans trop perturber les animaux.

L'oxyde de chrome est dosé suivant la technique mise au point par Françors et al. (1978). La matière organique est détruite et l'oxyde de chrome attaqué et oxydé par le mélange nitro-perchlorique en une seule opération. Une courte ébullition en présence de persulfate, qui assure l'oxydation complète du chrome, est suivie du titrage direct par le sel de Mohr, en présence de diphénylamine sulfonée.

En se basant sur les deux équations :

$$
\mathrm{MSE}=\frac{100 \mathrm{i}}{\mathrm{C}} \quad \text { et } \quad \mathrm{MSI}=\frac{\mathrm{MSE}}{1-\mathrm{DMS}}
$$

où :

MSE = matière sèche excrétée $(\mathrm{kg})$,

MSI = matière sèche ingérée $(\mathrm{kg})$,

$\mathrm{i}=$ dose journalière du chrome administrée $(\mathrm{g})$,

$\mathrm{C}=$ concentration fécale en $\mathrm{Cr}_{2} \mathrm{O}_{3}$,

DMS $=$ digestibilité de la matière sèche,

(2) Ce nouvel apport a nécessité un réequilibrage de la complémentation en grain, basé sur l'équivalence de l'apport énergétique global des compléments.

(3) Grande souplesse de l'animal, postérieur pointu et organe mammaire très développé. 
et en effectuant l'analyse de la digestibilité de la ration, on évalue la quantité de matière sèche totale ingérée par jour.

\section{a) Digestibilité de la ration}

L'analyse de la ration est faite par la méthode des composantes prises individuellement et non pas par celle des composantes associées. La raison en est la nécessité d'utiliser différents types d'équations de digestibilité, suivant que la composante est considérée comme un fourrage de type ligneux ou de type herbacé.

Divers auteurs proposent des équations de régression permettant de prédire la digestibilité in vivo de la MS analysée, la plus courante d'entre elles étant l'équation de Tilley \& Terry (1963) ; elle sera utilisée pour les complémentations en concentrés. Seul Nastis (1977) a étudié le cas de rations à base de chêne distribué à des caprins. Son étude est orientée vers le problème principal posé par la consommation de ce type de fourrage, à savoir, la présence et l'influence sur la digestibilité d'un certain pourcentage de tanins (acides phénoliques). Comme une teneur de 8 à 9 p. 100 de tanins (p. 100 MS en équivalents acides tanniques) semble caractériser le feuillage de chêne (50 p. 100 de la ration), l'équation proposée par cet auteur pour les espèces présentant plus de 1 p. 100 de tanins sera utilisée dans le cas des espèces ligneuses (75 p. 100 de la ration). Pour les espèces herbacées, nous utiliserons l'équation proposée pour des chèvres laitières par GIger et al. (1979) dans le cas de l'analyse des composantes individuelles.

$$
\begin{aligned}
& \text { Tilley \& Terry (1963) : DMD }=0,99 \text { IVD }-1,01 \text {. } \\
& \text { NASTIS (1977) : DMD }=0,40 \text { IVD }+29,1 . \\
& \text { GIGER et al. (1979) : } \mathrm{DMD}=0,72 \mathrm{IVD}+16,6 .
\end{aligned}
$$

\section{b) Analyses fourragères}

Seules ont été réalisées les analyses élémentaires :

- Matières azotées totales (méthode Kjeldahl).

- Cellulose (méthode Kurschner et Hannack, modifiée THILl, 1978).

- Digestibilité in vitro (technique Tilley et Terry réalisée avec du jus de rumen de moutons nourris au foin de prairie).

\section{c) Valeur énergétique}

L'énergie brute de la ration a été mesurée à la bombe calorimétrique. L'estimation de la valeur énergétique nette a été effectuée en utilisant les équations proposées par VAN Es (1975). Les matières azotées digestibles ont été calculées à partir des données de NASTIS \& MALEcheK (1981) pour le cas d'une ration distribuée en août à des caprins présentant une digestibilité de la MS semblable. Cette ration, composée de 80 p. 100 de chêne, a montré une digestibilité apparente de l'azote de 48,6 p. 100. 


\section{E. Comparaison des estimations des quantités de matière sèche ingérée}

La quantité de matière sèche ingérée distribuée en complément à l'étable est pesée, l'absence de pertes et de refus est contrôlée. De cette manière, on pourra également par la méthode de l'oxyde de chrome, évaluer la MS ingérée uniquement sur parcours. En effet, comme il n'est distribué que $0,35 \mathrm{~kg} \mathrm{MS} /$ jour de complément en moyenne, avec une digestibilité moyenne de 80 p. 100, on supposera satisfaisante l'approximation suivante :

avec :

$$
\mathrm{MSI}_{\mathrm{p}}=\frac{\mathrm{MSE}_{\mathrm{t}}-\mathrm{MSE}_{\mathrm{c}}}{1-\mathrm{DMS}_{\mathrm{p}}}
$$

$$
\begin{aligned}
& \mathrm{p}=\text { parcours, } \\
& \mathrm{c}=\text { complément, } \\
& \mathrm{t}=\text { total. }
\end{aligned}
$$

Un dernier terme de comparaison pourrait être apporté par la méthode du calcul des besoins zootechniques. Cependant, le nombre et l'importance des incertitudes qui pèsent sur le calcul des besoins ont conduit à ne pas utiliser cette méthode. Tout d'abord, une période expérimentale relativement courte fournit des résultats qu'il est difficile de mettre en relation avec des enregistrements zootechniques mensuels. Ensuite, la non-connaissance de l'importance que peuvent prendre les besoins supplémentaires liés aux dépenses énergétiques sur parcours ( 20 à 80 p. 100 des besoins d'entretien selon les cas, suivant divers auteurs) rend très délicate l'estimation de la couverture des besoins théoriques par l'ingéré.

\section{Résultats}

A. Composition de la ration estimée par la méthode des coups de dents et calcul de sa valeur nutritive

En période estivale (juillet et août), la ration des chèvres sur ce type de parcours est essentiellement à base de chêne blanc (Quercus pubescens) et de cornouiller sanguin (Cornus sanguinea). Vis-à-vis de ces espèces dominantes dans la ration, le comportement alimentaire des chèvres présente peu de variations individuelles importantes (respectivement 27 et 43 p. 100 de C.V., voir tableau 1).

La composition chimique des espèces démontre leur forte teneur moyenne en matière sèche et en cellulose (tabl. 2). La digestibilité du chêne blanc est beaucoup plus médiocre que celle du cornouiller ou de la psoralée bitumineuse. En moyenne, la qualité de la ration consommée sur parcours, recalculée à partir de ces données, est faible tant sur le plan énergétique qu'azoté (tabl. 3).

\section{B. Détermination des courbes d'excrétion fécale du marqueur oxyde de chrome}

C'est la détermination de la concentration moyenne quotidienne du marqueur dans les matières fécales prélevées qui permet l'estimation de la quantité de matière sèche ingérée. Cette concentration moyenne est établie à partir des courbes de l'excrétion horaire individuelle des 5 animaux témoins (fig. 1). 


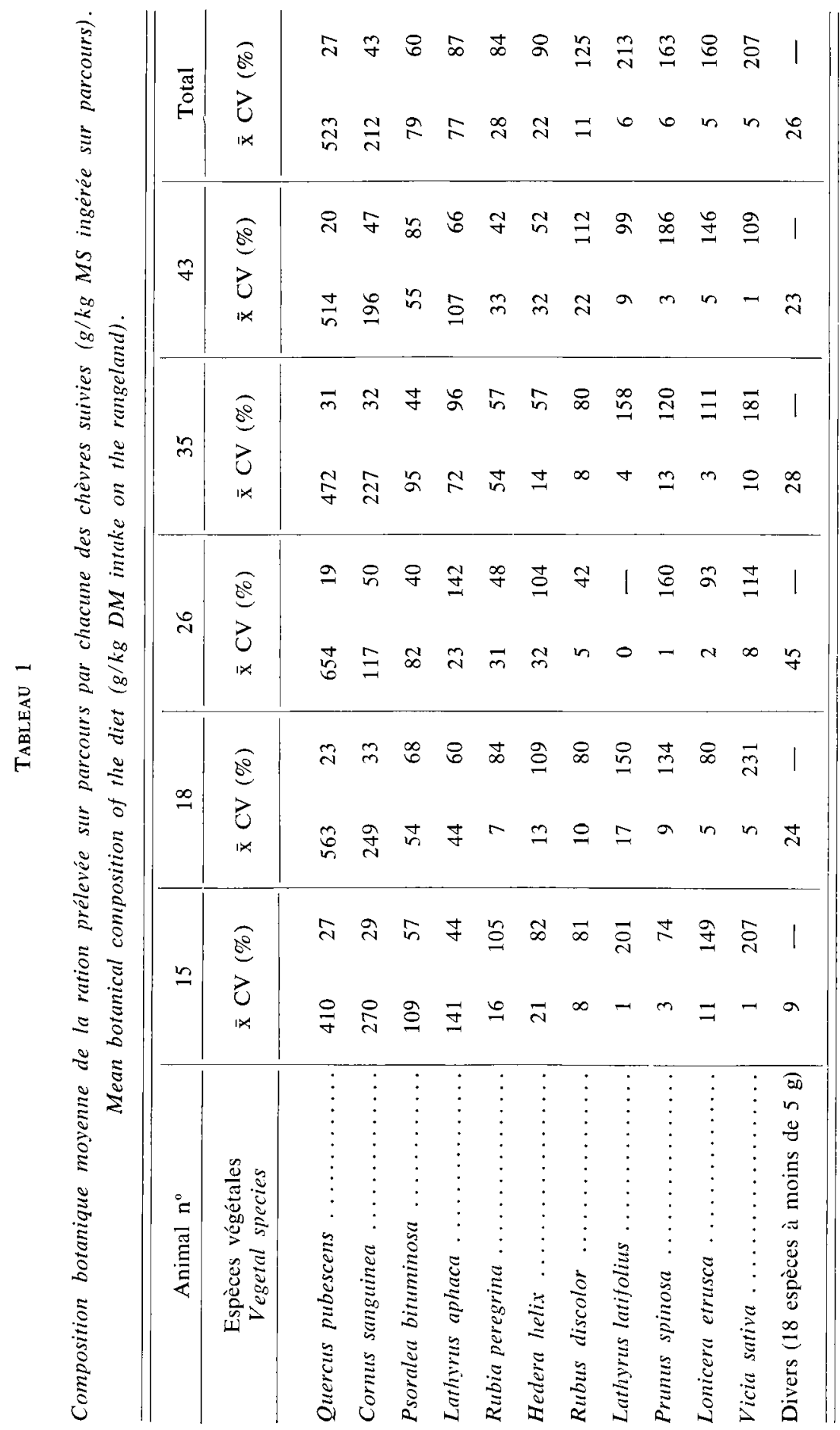




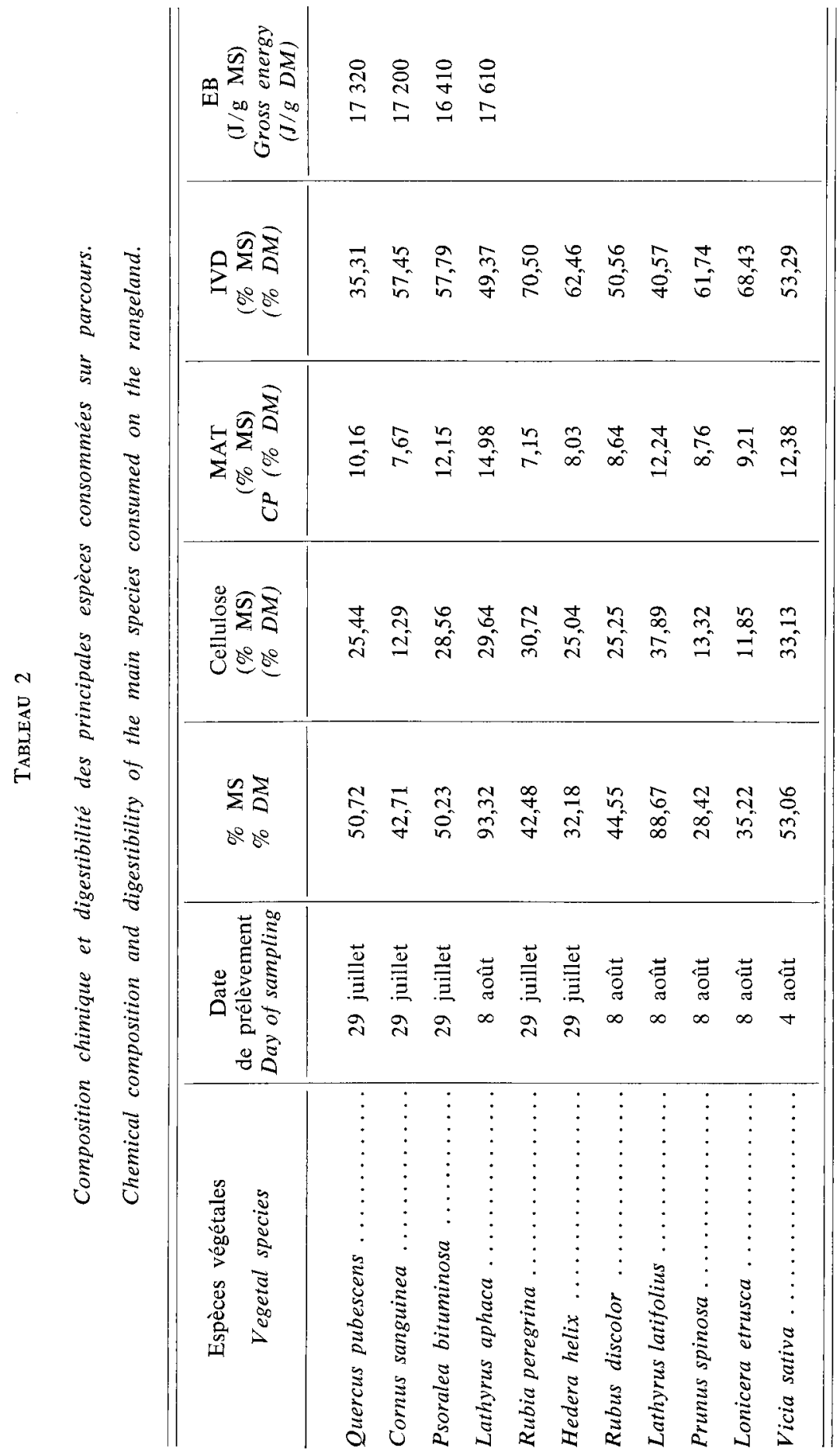




\section{TABleaU 3}

Valeur nutritive calculée de la ration ingérée sur le parcours.

Calculated nutritional yalue of the diet ingested on rangeland.

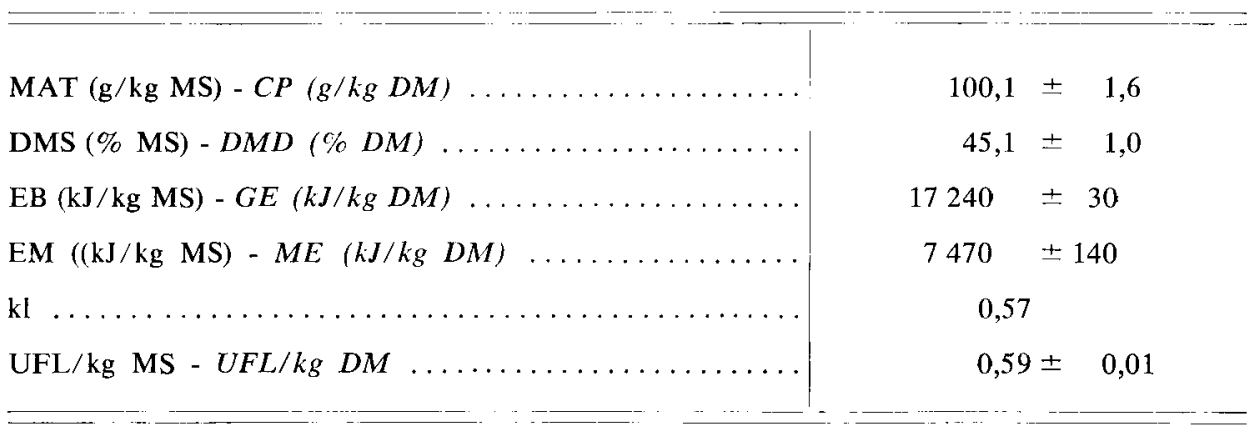

C. Performances zootechniques des animaux suivis

Les animaux étant en milieu de lactation, les taux butyreux et azoté du lait produit sont à un niveau particulièrement bas (tabl. 4).

D. Comparaison des estimations des quantités de matière sèche ingérée

La comparaison des estimations de MS ingérée sur parcours montre que la méthode d'observation directe des coups de dents propose une valeur supérieure en moyenne de 21 p. 100 à celle de la méthode du marqueur oxyde de chrome : 2,61 contre $2,16 \mathrm{~kg} \mathrm{MSI} /$ jour (tabl. 5). La moyenne des estimations, rapportée à un animal standard de $100 \mathrm{~kg}$ de poids vif, est de $4,6 \mathrm{~kg}$ MSI/jour. Ce résultat est en accord avec les données de Mac Kenzie (1956) et de Sauvant \& Morand-Fehr (1981) obtenues pour des animaux semblables en gabarit et en production.

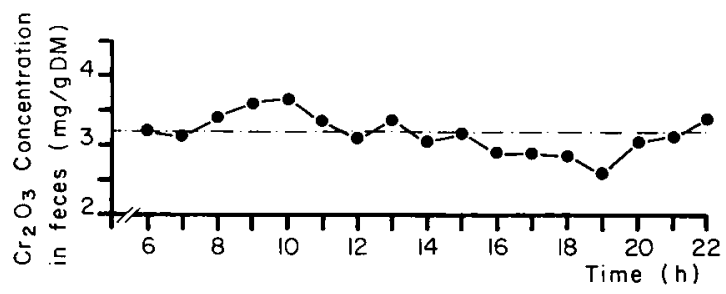

FIG. 1

Evolution chez le caprin de la concentration fécale moyenne en $\mathrm{Cr}_{2} \mathrm{O}_{3}$ au cours du temps (moyenne calculée à partir des concentrations horaires pour 5 animaux considérés pendant 6 jours).

Diurnal excretion cycle of chromic oxide by grazing goat on brushland. 
CONSOMMATION DE CHẼVRES SUR PARCOURS

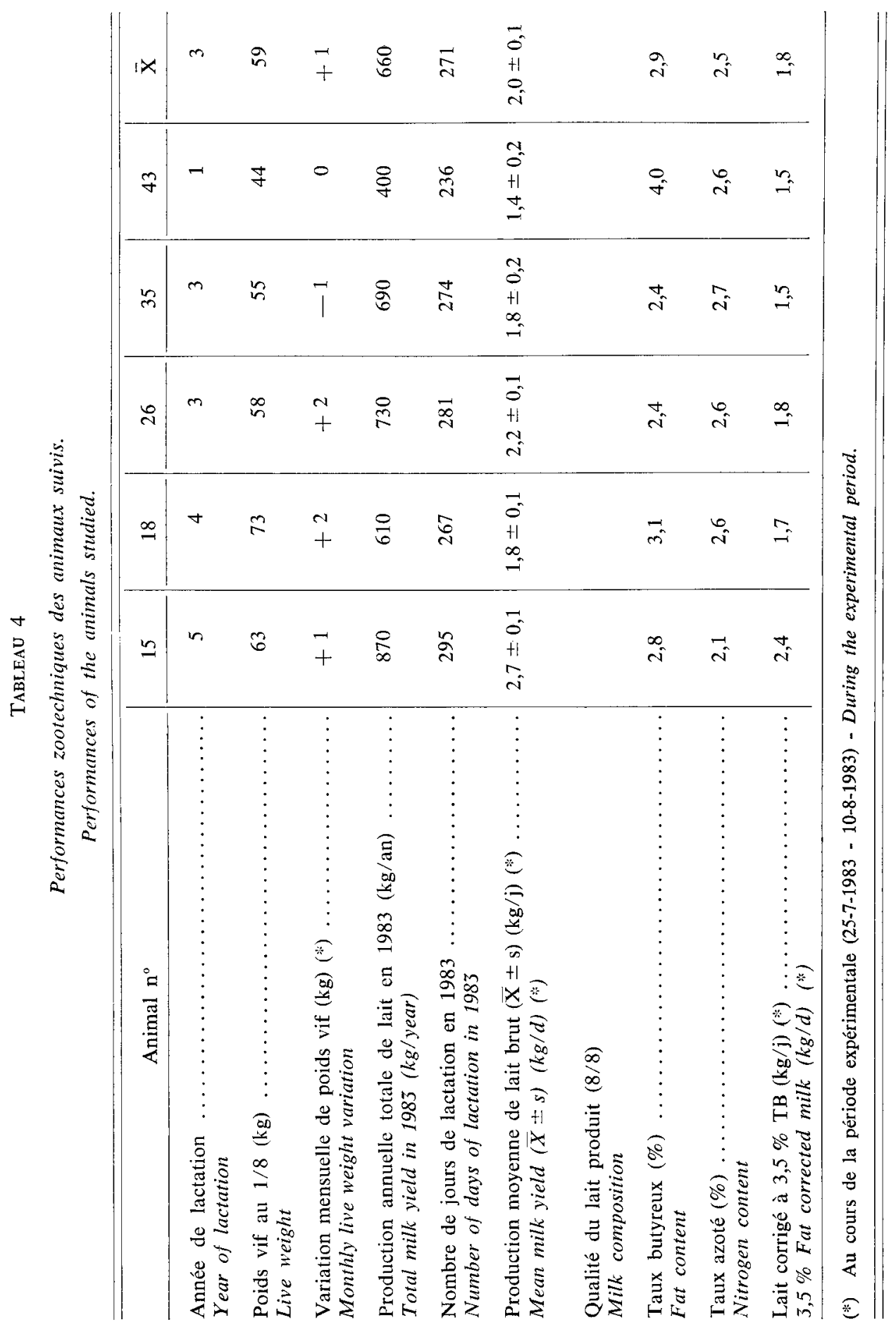




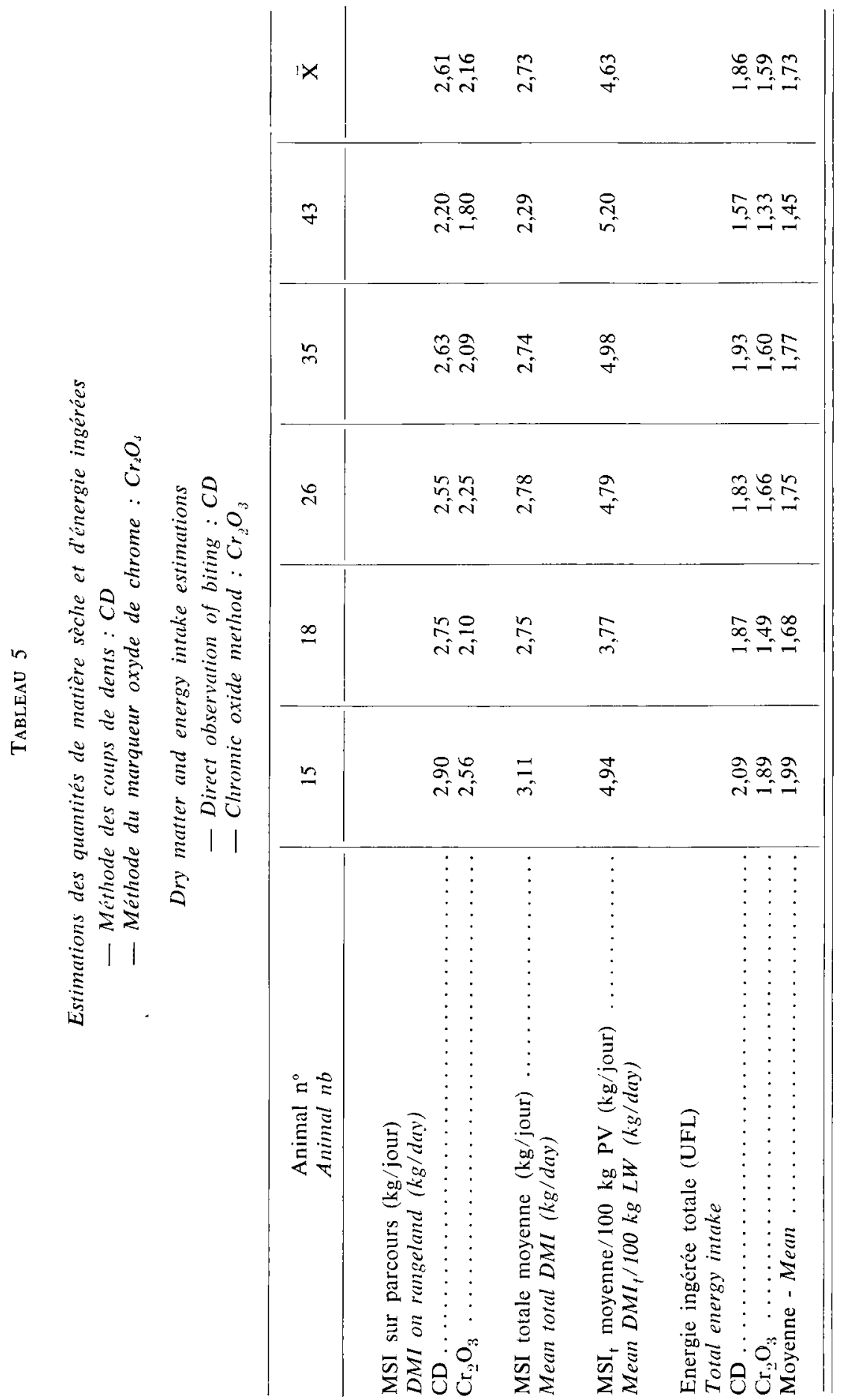


Les quantités d'énergie ingérées, exprimées en UFL et relatives à la ration totale (parcours et compléments distribućs à l'étable) sont en moyenne respectivement de 1,86 et 1,59 UFL/jour pour les méthodes des coups de dents et de l'oxyde de chrome.

\section{Discussion}

\section{A. Méthode d'observation directe des coups de dents}

La précision de la méthode dépend de la façon dont sont surmontées trois difficultés principales :

- la définition et la mesure du temps de pâturage,

- la mesure de la fréquence de coups de dents et la détermination de l'espèce consomméc,

- l'évaluation de la quantité ingérée par un coup de dents porté sur une espèce végétale donnée.

La durée de pâturage et la durée réelle des relevés sont chronométrées individuellement par l'ensemble des expérimentateurs. De plus, une personne supplémentaire est chargée de coordonner l'ensemble des activités et de tester la validité du chronométrage individuel.

Le choix d'effectucr les relevés sur l'ensemble des deux types d'activité (pâturage vrai et pâturage-déplacement) réduit considérablement les risques d'erreur dus à une mauvaise interprétation des temps d'activité. A l'aide d'un relevé supplémentaire de la fréquence de coups de dents, effectué en continu sur un animal durant les $7 \mathrm{~h}$ de sortic, on estime l'erreur commise par le relevé systématique de $10 \mathrm{mi}$ nutes toutes les 30 minutes de pâturage. En comparant le résultat obtenu par le relevé en continu avec ceux qui auraient été obtenu par des relevés systématiques, on obtient un écart relatif de 10 p. 100. Cet écart est jugé satisfaisant.

Le risque de mauvaise interprétation de la fréquence de coups de dents est estimé par une séric de tests collectifs. Dans le plus mauvais des cas, on obtient un écart à la moyenne inférieur à 5 p. 100. Cette faible valeur est justifiée par le fait que généralement le caprin ne prélève qu'une espèce à la fois. Ainsi, on estime à moins de 0,5 p. 100 du nombre total des coups de dents observés, les cas de bouchées composites ou de celles où l'espèce végétale n'a pu être déterminée.

La difficulté principale de la méthode réside dans l'estimation du «poids du coup de dents ». On a essayé de réduire les risques d'erreur d'estimation en effectuant la majorité des relevés à l'aide de distribution et de pesées sur balance de terrain placée sur le site de consommation. Malgré cela, une certaine hétérogénéité naturelle des coups de dents subsiste, et peut être illustrée par les résultats obtenus avec le chêne blanc ( 50 p. 100 de la ration).

Suite à la pesée de près de 2000 coups de dents sur chêne, on obtient un poids de coup de dents moyen de $1,28 \pm 0,40$ g MS (C.V. $=31$ p. 100).

Dans un site un peu différent dans la Drôme, mais dans le cas du chêne blanc également, Bouttier (résultats non publiés) mesure une valeur beaucoup plus faible 
du PCD $(0,69 \pm 0,12 \mathrm{~g}$ MS) pour une période moyenne de mai à août $(\mathrm{n}=400)$. Sur chêne vert, avec des animaux beaucoup plus légers, Bourbouze (1980) trouve en mai un PCD de $0,35 \mathrm{~g}$ MS $(\mathrm{n}=200)$ sur des parcours semi-arides marocains.

Toujours sur chêne vert, avec le troupeau ardéchois présenté ici, Meuret (résultats non publiés) trouve en avril un PCD de $0,87 \pm 0,06 \mathrm{~g} \mathrm{MS}(\mathbf{n}=300)$. La valeur du coup de dents est donc étroitement liée à un site, à une saison et à un type de troupeau.

Dans le cas du chêne (consommé à près de $1,4 \mathrm{~kg} \mathrm{MS} / \mathrm{jour}$ ), notons qu'une variation du PCD de 31 p. 100 conduit à une variation de la MSI de l'ordre de $0,4 \mathrm{~kg}$ MS/jour.

On peut tester l'influence de quelques variables sur le poids du coup de dents porté sur le chêne, par exemple (dans le cas de 160 rameaux de chêne distribués) :

A :

$\mathrm{Y}=$ poids frais du coup de dents moyen $(\mathrm{g})$

$\mathrm{X}=$ poids vif de l'animal le $1-8-1983(\mathrm{~kg})$ (les relevés sont nominatifs)

$$
\begin{aligned}
& \mathrm{Y}=1,67+0,02 \mathrm{X} \\
& \mathrm{n}=160 ; \mathrm{r}=0,17
\end{aligned}
$$

B :

$\mathrm{Y}=$ poids frais du coup de dents moyen $(\mathrm{g})$

$\mathrm{X}=$ production moyenne hebdomadaire de lait brut $(1 / 8-7 / 8)(\mathrm{kg})$

$\mathrm{Y}=2,04+0,31 \mathrm{X}$

$\mathrm{n}=160 ; \mathrm{r}=0,30$

C :

$\mathrm{Y}=$ poids frais du coup de dents moyen $(\mathrm{g})$

$\mathrm{X}=\frac{\text { nombre de coups de dents portés sur le rameau }}{\text { poids frais initial du rameau }}\left(\mathrm{g}^{-1}\right)$

(tous les rameaux sont coupés à la section : diamètre $=1 \mathrm{~cm}$ )

$\mathrm{Y}=3,81-7,29 \mathrm{X}$

$\mathrm{n}=160 ; \mathrm{r}=-0,59$

Malgré le niveau non-significatif des corrélations obtenues, il semble que la variation du PCD dans le cas du caprin broutant des arbustes soit plus influencée par la morphologie de l'arbuste à brouter (présentation du «disponible ») que par les besoins zootechniques de l'animal.

En ce qui concerne les poids de coup de dents estimés par la méthode du prélèvement simulé, d'après les résultats obtenus par l'ensemble des expérimentateurs, l'écart maximum observé a été de près de 20 p. 100. Comme il est impossible dans ce cas de dégager les parts relatives à l'erreur expérimentale et à la variabilité naturelle du prélèvement, il est conseillé de limiter autant que possible le nombre des estimations par simulation, et cela d'autant plus que la végétation herbacée est dense. 
B. Méthode du marqueur oxyde de chrome

\section{Excrétion fécale du marqueur}

La figure 1 décrit l'évolution journalière de la moyenne générale de la concentration du $\mathrm{Cr}_{2} \mathrm{O}_{3}$ dans les fèces prélevées. Cette moyenne est pondérée suivant le nombre des relevés effectués chaque jour. Un résultat moyen obtenu sur tous les animaux dépend de deux facteurs : le facteur « jour» et le facteur « animal ». Cette observation a justifié le traitement statistique des résultats dans le but de dégager l'évolution journalière des composantes de la variance générale. Ces composantes sont la variance entre les jours $\left(\sigma_{d}^{2}\right)$ et la variance entre les animaux $\left(\sigma_{\mathfrak{a}}^{2}\right)$. C'est le résultat de ce traitement qui est indiqué à la figure 2 .

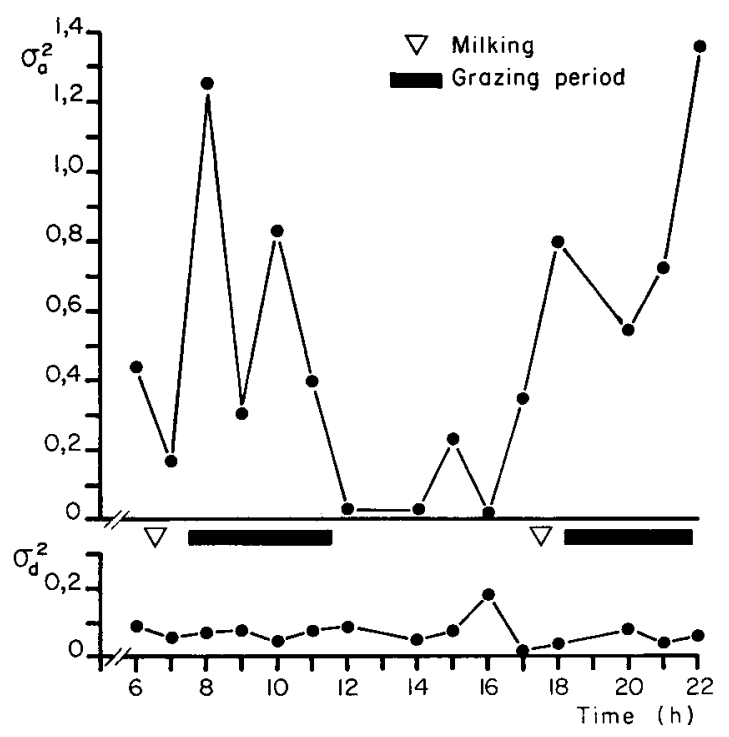

FIGURE 2

Evolution chez le caprin des composanies de la variance de l'excrétion fécale $\mathrm{du}_{\mathrm{Cr}} \mathrm{Cr}_{3}$ chtre les animaux $\left(\sigma_{a}^{2}\right)$ et entre les jours $\left(\sigma_{a}^{2}\right)$.

Evolution of the variance components of the $\mathrm{Cr}_{2} \mathrm{O}_{3}$ faecal excretion between animals $\left(\sigma_{a}^{2}\right)$ and between days $\left(\sigma_{d}^{2}\right)$.

On peut y observer que l'évolution journalière des composantes de la variance entre animaux est très importante ${ }^{(4)}$, ce qui peut être intéressant dans le cas d'études ultérieures visant à mettre en évidence des différences dans le comportement alimentaire des animaux. Ce type de variance est particulièrement élevé en période de sortie sur parcours, mais il tend à se stabiliser en période de repos à l'étable. On peut également observer que l'évolution des composantes de la variance entre jours est faible (5), et non significativement dépendante des périodes de sortie.

(4) $\sigma_{\mathfrak{a}}^{2}$ varie de 0,02 à 1,38 .

(5) $\sigma_{d}^{2}$ varie de 0,01 à 0,18 . 
Ces observations conduisent à proposer que, dans le cas d'un travail futur, le nombre des animaux marqués soit multiplié (par exemple par un facteur 2). Il ne semble, par ailleurs, pas nécessaire de multiplier le nombre des jours de relevé, ce dernier pouvant, au contraire, être réduit.

L'analyse des courbes d'excrétion fécale met en évidence une certaine périodicité de la concentration fécale en $\mathrm{Cr}_{2} \mathrm{O}_{33}$. Cela rend possible la proposition d'un schéma d'échantillonnage réduit, ne nécessitant que deux prélèvements par jour, effectués de préférence durant les heures de stabulation (tabl. 6). Cette proposition n'est bien entendu applicable qu'à un horaire estival de sortie et où l'administration du marqueur s'effectue à $6 \mathrm{~h}$ et $17 \mathrm{~h}$.

\section{TABleAu 6}

Processus simplifié d'échantillonnage des fèces sur parcours (sorties estivales).

Twice daily sampling of faeces method on rangeland (summer grazing).

\begin{tabular}{c|c|c}
\hline \hline $\begin{array}{c}\text { Heures } \\
\text { Hours }\end{array}$ & $\begin{array}{c}\text { Ecart moyen (1) } \\
\overline{\mathrm{x}}_{1}-\overline{\mathrm{x}}_{\mathbf{0}}\end{array}$ \\
\hline $6-12$ & $-0,02$ & $\begin{array}{c}\text { Ecart-type (2) } \\
\text { Standard deviation }\end{array}$ \\
$6-13$ & 0,06 & 0,45 \\
$6-14$ & $-0,07$ & 0,46 \\
$6-15$ & $-0,01$ & 0,39 \\
$12-13$ & 0,02 & 0,40 \\
$12-15$ & $-0,04$ & 0,70 \\
$13-14$ & $-0,03$ & 0,49 \\
$13-15$ & 0,03 & 0,62 \\
\hline
\end{tabular}

Deux échantillons par jour à l'étable :

Optimum $: 6 \mathrm{~h}-13 \mathrm{~h} 30:$ Ecart-type $=0,43-$ Ecart moyen $\rightarrow 0$.

Two samplings per day at the shed:

Better way: $6 \mathrm{~h}-13 \mathrm{~h} 30:$ Standard deviation $=0.43-\bar{x}_{c}-\bar{x}_{0} \rightarrow 0$.

(1) Ecart moyen : $\left(\overline{\mathrm{x}}_{\mathrm{e}}-\overline{\mathrm{x}}_{0}\right)=$ écart entre la moyenne calculée à partir de l'cnsemble des obscrvations pendant $16 \mathrm{~h}$, et la moyenne observée.

$\left(x_{c}-x_{b}\right)=$ mean difference between the calculated mean $\left(x_{t}\right)$, from the results during a $16 \mathrm{~h}$ period, and the observed mean $\left(\bar{x}_{0}\right)$.

(2) Ecart-type = écart-type des écarts entre la moyenne calculée sur l'ensemble des observations pendant $16 \mathrm{~h}$ pour chaque animal et la moyenne générale des échantillons.

Dans le cas d'horaires significativement différents, il est probable que les courbes d'excrétion fécale soient différentes.

Pour obtenir un échantillonnage adéquat, il est nécessaire que l'écart-type et l'écart moyen (en valeur absolue) soient minimum. Cette condition est remplie si les prélèvements ont lieu à $6 \mathrm{~h}$ et entre $13 \mathrm{~h}$ et $14 \mathrm{~h}$, car dans ce cas l'écart moyen, en variant de 0,06 à $-0,07$, peut s'annuler (tabl. 6). 


\section{Estimation des quantités ingérées}

La composition particulière de la ration induit deux incertitudes dans l'application de la méthode du marqueur oxyde de chrome, d'une part en ce qui concerne le dosage du marqueur dans les matières fécales, et d'autre part lors de l'estimation de la digestibilité réelle de la ration.

Si la méthode de dosage du marqueur ne pose plus de problème, suite aux travaux de FrançoIs et al. (1978), elle est perturbée dans ce cas-ci par la présence au sein de la ration de certains éléments chimiques réducteurs ( $\mathrm{Mn}$ et Fe par exemple) non habituellement signalés en des proportions semblables dans les fourrages plus classiques. Ces éléments réducteurs provoquent une surestimation de la concentration en $\mathrm{Cr}_{2} \mathrm{O}_{3}$ : il est donc nécessaire d'effectuer une correction moyenne par calibrage de titration, en faisant l'hypothèse de l'excrétion régulière de ces éléments.

Mais l'incertitude majeure provient de l'estimation de la digestibilité réelle de la ration. WiLSON et al. (1971) ont affirmé que les techniques in vitro communément utilisées tendent à sous-estimer la digestibilité des rations de qualité médiocre et à prédominance de ligneux. Devendra (1978) suggère que les chèvres ont une grande capacité à digérer les fibres brutes, particulièrement dans le cas des rations de qualité médiocre et à haute teneur: en fibres. Morand-Fenr (1981), au cours d'une étude bibliographique, observe une meilleure digestibilité de la cellulose chez la chèvre par rapport au mouton à mesure que la teneur en cellulose brute du fourrage atıgmente, et l'inverse à mesure que la teneur en matières azotées du fourrage augmente. D'autre part, Huston \& Silelton (1967) observent que la rétention protéique augmente chez la chèvre quand on inclut des feuilles de chêne dans le régime. WiLson et al. (1975) confirment ces résultats en observant une meilleure utilisation de l'azote chez la chèvre par rapport at mouton. Tout récemment, Nastis (communication personnelle) étudiant la digestibilité apparente de rations à base de chêne kermès (Quercus coccifera) distribuées à de petites chèvres grecques détermine une équation de régression (DMD $=0,69$ IVD $+34,6 ; \mathrm{r}^{2}=0,76$ ) bien plus favorable à la digestibilité in vivo que les équations généralement utilisées.

Ces quelques remarques laissent à penser qu'il est très délicat d'appliquer à la chèvre au pâturage sur parcours les différents modèles d'alimentation élaborés pour d'autres ruminants, et que dès lor's, il est fort probable que la valeur nutritive de la ration ingérée sur parcours soit sous-estimée, ainsi que le niveau de consommation de la MS sur parcours dans le cas de l'utilisation de la méthode du marqueur oxyde de chrome.

\section{Méthode des besoins théoriques}

Comme cela a déjà été dit at paragraphe II-E, l'estimation de l'apport alimentaire du parcours par la méthode du calcul des besoins théoriques n'a pas été réalisée, à cause des trop nombreuses incertitudes qui subsistent.

D'une part, les recommandations pour les besoins d'entretien s'applfquent à des animaux élevés en chèvrerie, ou ayant peu d'activité; elles doivent être majorées pour des chèvres conduites sur parcours d'une valeur dépendant de leur niveau d'activité journalière (French, 1944; Lindahl, 1974). BlAXTER (1962) propose des valeurs de dépenses énergétiques nécessaires par $\mathrm{km}$ de terrain plat et par $100 \mathrm{~m}$ de dénivellation. L'application de ces valeurs à un parcours relativement plat mais 
extrêmement accidenté (un plateau calcaire formé en lapiaz) est trop incertaine pour être jugée satisfaisante.

D’autre part, une période expérimentale relativement courte (6 jours) fournit des résultats difficiles à mettre en relation avec les enregistrements zootechniques portant sur une période plus longue. En effet, on peut estimer que d'assez bonnes corrélations sont obtenues entre la moyenne des estimations de la MS ingérée totale quotidienne (Y : $\mathrm{kg} /$ jour) et les enregistrement de la production laitière $(\mathrm{X}$ : $\mathrm{kg}$ /jour) :

$$
\begin{aligned}
& X=\text { Production de lait brut } \quad \rightarrow Y=1,64+0,55 X ; r=0,93 \\
& \mathrm{n}=30 \\
& \mathrm{X}=\text { Production de lait à } 3,5 \mathrm{p} .100 \mathrm{~TB} \rightarrow \mathrm{Y}=1,58+0,65 \mathrm{X} ; \mathrm{r}=0,82 \\
& \mathrm{n}=30
\end{aligned}
$$

La corrélation obtenue entre la moyenne des estimations de la MS ingérée totale quotidienne ( $\mathrm{Y}: \mathrm{kg}$ /jour) et le poids vif enregistré la veille du premier jour de suivi (X : $\mathrm{kg} P V$ ) reste juste satisfaisante, mis à part le nombre insuffisant de donnćes :

$$
\begin{aligned}
\mathrm{Y}=1,66+0,02 X ; & \mathrm{r}=0,67 \\
\mathrm{n} & =5
\end{aligned}
$$

Par contre, si l'on tente de relier le taux de couverture des besoins énergétiques totaux (p. 100) (besoins d'entretien majorés arbitrairement de $30 \mathrm{p} .100$ ) avec les variations mensuelles de PV corrigé enregistrées au cours de deux pesées à intervalle de 28 jours, il apparaît que ce sont les animaux qui ont un excédent théorique d'énergie qui perdent du poids ou restent à poids constant, alors que les animaux dont les besoins théoriques sont au mieux satisfaits, ou en déficit, gagnent du poids.

Malgré l'insuffisance du nombre des pesées, il nous semble possible de confirmer ici le doute souvent émis quant à l'origine physiologique des variations de poids enregistrées dans le cas de caprins sur parcours. A ce propos, pour les cas où des mesures ponctuelles du niveau d'alimentation sont à mettre en relation avec des enregistrements mensuels des performances, les études visant à obtenir une estimation du bilan énergétique sur des périodes expérimentales plus courtes sans avoir à estimer l'évolution pondérale des animaux sont à encourager (études sur les phénomènes de mobilisation des réserves corporelles chez le caprin : Giger \& SAuvant, 1982).

\section{Conclusion}

L'application des deux méthodes d'évaluation de la quantité de matière sèche ingérée sur parcours par des chèvres confirme la nécessité de poursuivre les recherches sur ce type particulier de pâturage. Lors de la présente étude, de nombreuses incertitudes sont apparues : elles sont liées au peu d'informations existantes relatives au sujet traité.

Les estimations des quantités ingérées totales sont respectivement, pour la méthode d'observation directe des coups de dents et pour la méthode oxyde de chrome, 
de 2,61 et $2,16 \mathrm{~kg}$ MS/animal . jour. Ces résultats montrent qu'une utilisation importante du parcours (82 p. 100 des apports énergétiques totaux) liée à une faible valeur alimentaire de la ration $(0,59 \mathrm{UFL} / \mathrm{kg} \mathrm{MS})$ conduit à un niveau de consommation totale quotidienne élevée (voisine de $2,7 \mathrm{~kg}$ MS pour un animal de $60 \mathrm{~kg}$ PV).

L'estimation du taux de couverture des besoins théoriques par l'ingéré n'a pas pu être réalisée. En effet, trop d'incertitudes demeurent quant à, d'une part, l'origine physiologique des variations de poids enregistrées, et d'autre part, l'importance des besoins d'entretien supplémentaires liés aux déplacements sur parcours.

En ce qui concerne la méthode d'observation directe des coups de dents, une étude de la fiabilité et de la reproductibilité des mesures de la fréquence de coups de dents dans divers milieux pâturés semble nécessaire. Il serait également intéressant d'étudier la variabilité naturelle des «poids du coup de dents", et d'aboutir ainsi à une modélisation du prélèvement suivant le type animal, l'espèce végétale, son stade phénologique et la nature du milieu pâturé.

L'application de la méthode du marqueur oxyde de chrome met en évidence la nécessité d'une analyse de la digestibilité apparente des fourrages ligneux, en relation avec la présence de certains constituants secondaires, tels que les tanins. Une étude en ce sens pourrait être envisagée en conditions contrôlées, avec utilisation du marqueur oxyde de chrome.

L'examen de l'excrétion fécale du marqueur oxyde de chrome sur parcours doit être conduit attentivement. L'intérêt de l'utilisation du marqueur, dans le but d'étudier les variations individuelles d'ingestion et de comportement alimentaire, est confirmé par l'analyse des composantes de la variance entre les animaux de l'excrétion fécale de ce marqueur.

Pour conclure, il nous semble important d'insister sur le fait que les deux méthodes utilisées, bien qu'elles visent un même objectif, ne sont pas entièrement substituables. En particulier, dans le cas d'un pâturage hétérogène, la méthode d'observation directe des coups de dents fournit à la méthode du marqueur la donnée indispensable relative à la composition en poids sec de la ration. De plus, relativement complémentaires, ces deux méthodes permettent l'étude de facteurs de variation différents : l'effet du milieu, de sa composition botanique, du mode de conduite du troupeau et du jour pour la méthode des coups de dents; l'effet moyen de la période et du type d'animal pour la méthode du marqueur indigestible.

Reçu en septembre 1984.

Accepté en avril 1985.

\section{Summary \\ Feed intake of dairy goats on rangelands \\ - Direct observation of biting method \\ - Chromic oxide method}

Two methods for estimating the DM intake of goats grazing on rangeland were studied. They were simultaneously applied to 5 Alpine Chamoisée dairy goats for 6 days. The animals grazed a white oak brushwood (Quercus pubescens) on the calcarcous plateau near 
the Ardèche river (Southern France). During the study (July 25 to August 10, 1983) the flock took 82 p. 100 of its energy requirements on the rangeland.

The first method of estimation was based on the observation of grazing behaviour and the rate of biting. An evaluation of the "weight of bites 》 and that for each species consumed allowed to estimate the dry matter intake during the grazing period. In that study, evaluation of the weight of bites on the ligneous species ( 75 p. 100 of the diet : see table 1) used a particular method : distribution of branches and accurate weighing on the grazing site.

The second method used chromic oxide $\left(\mathrm{C}_{2}, \mathrm{O}_{:}\right)$as a marker incorporated to pelleted lucerne hay. The marker was ingested without losses or refusals. The method used to determine the $\mathrm{Cr}_{3} \mathrm{O}_{3}$ concentration was very rapid and precise : titration after an oxidation with a mixture of nitric and perchloric acids. A graph of the diurnal excretion cycle of chromic oxide by goats grazing a rangeland was established (fig. 2).

Feed intake was 2.61 and $2.16 \mathrm{~kg} \mathrm{DM} / \mathrm{animal} / \mathrm{day}$, respectively for the direct biting observation and for the chromic oxide method. The results show that a large utilization of rangelands bound to a low nutritional value of the ligneous diet $(0.59 \mathrm{MFU} / \mathrm{kg} \mathrm{DM})$ leads to a high daily feed intake (nearly $2.7 \mathrm{~kg}$ DM/day for an animal of $60 \mathrm{~kg} \mathrm{LW}$ ).

The application of these methods shows the existence of gaps related to lack a knowledge about the treated topic (true digestibility of the diet, supplement of maintenance requirements due to grazing activity). Discussion of the problems encountered emphasizes the necessity of carrying out further research on this kind of pasture.

Key words : goat, rangeland, dry matter intake, behaviour method, indicator technique chromic oxide, grazing behaviour.

\section{Rëférences bibliographiques}

AlLDEN W.G., WhitTraKer I.A., 1970. The determinant of herbage intake by grazing sheep : the interrelationship of factors influencing herbage intake and availability. Aust. J. Agric. Res., 21, 755-766.

Arnold G.W., Dudzinski M.L., 1967. Studies on the diet of the grazing animal. III. - The effect of pasture species and pasture structure on the herbage intake of sheep. Aust. J. Agric. Res., 18, 657-666.

BlaXter K.L., 1962. Energy feedings standards of ruminants. Hutchinson Scientific and Technical, London, 284 p.

Bourbouze A., 1980. Utilisation d'un parcours forestier pâturé par des caprins. Fourrages, 82, $121-144$.

Chacon E., Stobis T.H., Sandlands R.H., 1976. Estimation of herbage consumption by grazing cattle using measurements of eating behaviour. J. br. Grassl. Soc., 31, 81-88.

Currie P.O., Reichert D.W., Malecheck J.C., Wallmo O.C., 1977. Forage selection comparisons for mule deer and cattle under managed Ponderosa Pine. J. Range Manage., 30, 352-356.

Devendra C., 1978. The digestive efficiency of goats. World Rev. Anim. Prod., 14, 9-12.

François E., Thill N., Thewis A., 1978. Méthode rapide de dosage de l'oxyde de chrome dans les aliments, les fèces et les contenus digestifs par titrage après oxydation nitroperchlorique. Ann. Zootech., 27, 335-361.

French M.H., 1944. The feeding of goats. E. Afric. agric. J., 10, 66-71.

Giger S., Lila M., Sauvant D., Morand-Fehr P., 1979. In vitro prediction of whole ration digestibility. Ann. Zootech., 28, 373-379.

Giger S., Sauvant D., 1982. Utilisation des acides gras non estérifiés plasmatiques pour la prévision des bilans et besoins énergétiques de la chèvre en lactation. Ann. Zootech., 31, 149-160. 
GRIEGo R.R., 1975. Spring grazing and bioenergetic studies of sheep and goats. Tunisian presaharian project. Desert Biome Prog. Rep., n 3, Logan (Utah), 3-5.

Huston J.E., Shelton M., 1967. Nutritional requirements of the Angora goats. Tex. Agr. Exp. Sta., 2451, 32.

I.N.R.A., 1980. Alimentation des ruminants. Ed. I.N.R.A. Pub. (route de Saint-Cyr), 78000 Versailles, France, 621 p.

LE Du Y.L.P., Penning P.D.P., 1979. Advances in the indirect techniques to determine herbage intake. Proc. III European Grazing Workshop. (2-5 april 1979). Lelystad., 3-10.

LinDaHL I.L., 1974. Nutrition and feeding goats. In : Digestive Physiology and Nutrition of Ruminants. Church Ed., Distr. by O. and Boocks, Oregon, U.S.A., 261-275.

MaC Kenzie D., 1956. Goat Husbandry. Faber and Faber, Ltd., London, Ed., 350 p.

Meiss J.A.C., 1981. Herbage intake by grazing dairy cows. Agricultural Research Reports. Centre for agricultural publishing documentation, Wageningen (Netherlands), $263 \mathrm{p}$.

Morand-Fenr P., 1981. Caractérisation du comportement alimentaire et de la digestion des caprins. In : Nutrition et systèmes d'alimentation de la chèvre. Symposium International. Tours, France, 1, 21-45.

Nastis A.S., 1977. In : Malechek J.C., Provenza F.D., 1981. Fceding behaviour and nutrition on rangelands. In : Nutrition et systèmes d'alimentation de la chèvre. Symposium International. Tours, France, 1, 411-428.

Nastis A.S., MaLECheK J.C., 1981. Digestion and utilization in oak browse by goats. J. Anim. Sci., 53, 283-290.

NEFF D.J., 1974. Forage preference of trained mule deer on the Beavercreek watersheds. Arizona Game and Fish Dep. Spec. Rep., 4, 61 p.

Sauvant D., Morand-Fehr P., 1981. In : Morand-Fehr P., 1981. Caractéristiques comportementales et digestives. In : Nutrition et systèmes d'alimentation de la chèvre. Symposium International. Tours, France, 1, 21-45.

Stonbs T.H., 1975. The effect of plant structure on the intake of tropical pasture. III. - Influence of fertilizer nitrogen on the size of bite harvested by Jersey cows grazing Setaria anceps c.v. Kazungula swards. Aust. J. Agric. Res., 26, 997-1007.

ThILl N., 1978. Mise au point d'une technique de mesure simple et précise de la qualité et de la quantité de l'herbe consommée par le bovin au pâturage. Thèse de Doc. Fac. des Sc. Agron. de l'Etat. Gembloux (Belgique), $201 \mathrm{p}$.

Thill N., François E., Thewis A., Thielemans M.-F., 1978. Comparaison chez le mouton de l'oxyde de chrome-papier et du radiocérium, marqueurs de la phase solide des digesta. Ann. Zootech., 27, 363-376.

TIlley J.M.A., TerRy R.A., 1963. A two stage technique for the in vitro digestion of forage crops. J. Brit. Grassld. Soc., 18, 104-111.

Tissier M., Bechet G., Molenat G., 1975. Appareils de collecte totale des fèces pour agneaux en allaitement ou à l'engrais et pour brebis. Ann. Zootech., 24, 595-602.

VAn Es A.J.H., 1975. Feed evaluation for dairy cows. Livestock Prod. Sci., 2, 95-107.

Wilson A.D., Weir W.C., Torrel D.T., 1971. Comparison of methods of estimating the digestibility of range forage and browse. J. Anim. Sci., 32, 1042-1045.

Wilson A.D., Leich C.H., Hindley N.H., Mulham W.E., 1975. Comparison of the diets of goat and sheep on a Woodland Community in Western New South Wales (Australia). Aust. J. Exp. Agr. Anim. Husb., 15, 45-53. 